\title{
Event-Related Activation in the Human Amygdala Associates with Later Memory for Individual Emotional Experience
}

\author{
Turhan Canli, ${ }^{1}$ Zuo Zhao, ${ }^{1}$ James Brewer, ${ }^{1}$ John D. E. Gabrieli, ${ }^{1,2}$ and Larry Cahill ${ }^{3,4}$ \\ Departments of ${ }^{1}$ Psychology and ${ }^{2}$ Radiology, Stanford University, Stanford, California 94305, and ${ }^{3}$ Center for the \\ Neurobiology of Learning and Memory and ${ }^{4}$ Department of Neurobiology and Behavior, University of California, \\ Irvine, California 92697
}

\begin{abstract}
The role of the amygdala in enhancing declarative memory for emotional experiences has been investigated in a number of animal, patient, and brain imaging studies. Brain imaging studies, in particular, have found a correlation between amygdala activation during encoding and subsequent memory. Because of the design of these studies, it is unknown whether this correlation is based on individual differences between participants or within-subject variations in moment-to-moment amygdala activation related to individual stimuli. In this study, participants saw neutral and negative scenes and indicated how emotionally intense they found each scene. Separate functional magnetic resonance imaging responses in the amygdala for
\end{abstract}

each scene were related to the participants' report of their experience at study and to performance in an unexpected memory test 3 weeks after scanning. The amygdala had the greatest response to scenes rated as most emotionally intense. The degree of activity in the left amygdala during encoding was predictive of subsequent memory only for scenes rated as most emotionally intense. These findings support the view that amygdala activation reflects moment-to-moment subjective emotional experience and that this activation enhances memory in relation to the emotional intensity of an experience.

Key words: amygdala; affect; emotion; arousal; individual experience; memory
Emotional experiences are often better recalled than nonemotional ones (Christianson, 1992). For example, people show superior declarative memory for emotionally vivid segments of a story, relative to emotionally neutral segments (Cahill et al., 1995; Adolphs et al., 1997). The amygdala appears to play a critical role in such an enhancement of emotional memory, because patients with bilateral amygdala damage do not remember emotional better than neutral story segments (Cahill et al., 1995; Adolphs et al., 1997).

Imaging studies have shown that amygdala activation correlates with emotional memory in the intact brain. Two positron emission tomography (PET) studies (Cahill et al., 1996; Hamann et al., 1999) and one functional magnetic resonance imaging (fMRI) study (Canli et al., 1999) reported significant correlations between amygdala activation related to emotional stimuli and subsequent memory. In all three studies, individuals who showed strong amygdala activation in response to a set of emotional stimuli (relative to other study participants) also showed superior memory for those stimuli (relative to other study participants). Importantly, such correlations between amygdala activation and subsequent memory were not observed for emotionally neutral stimuli.

These first imaging studies have identified a correlation between amygdala activation and declarative memory for emotional stimuli across different individuals. This between-subjects study design allows for at least three alternative interpretations of the data. The first is that some individuals are more responsive to emotional experiences than others. The observed amygdala activation therefore would reflect a tonic personality characteristic. The second is that some individuals, during a particular scanning session, may have been in some sort of state that enhanced responsiveness to emotional experience. The third interpretation is that the amygdala is responsive in a dynamic or phasic way to

Received March 10, 2000; revised July 19, 2000; accepted July 21, 2000.

Correspondence should be addressed to Turhan Canli, Department of Psychology, Jordan Hall, Stanford, CA 94305. E-mail: canli@psych.stanford.edu.

Copyright $\odot 2000$ Society for Neuroscience $0270-6474 / 00 / 200001-05 \$ 15.00 / 0$ moment-to-moment individual emotional experience, so that amygdala activation would reflect a flexible, rapidly changing emotional response that ought to be observable within an individual. This explanation is consistent with animal studies (Cahill and McGaugh, 1990; McGaugh et al., 1996).

The phasic interpretation makes the specific prediction that the amygdala is sensitive to the emotional intensity of a stimulus, where intensity may refer to the valence or arousal characteristics of that stimulus, or a combination of both. The phasic interpretation makes the additional prediction that those emotionally intense stimuli that produce greater amygdala activation should be better remembered than stimuli that produce less amygdala activation.

The present study used event-related fMRI in a within-subject, subsequent-memory design (Brewer et al., 1998) to test the predictions made by the phasic interpretation. Participants saw neutral and negative scenes and indicated how they experienced the emotional intensity of each scene. A separate fMRI response was recorded in the amygdala for each such emotional experience. Three weeks later, participants' memories for the scenes were assessed. Thus, each amygdala response could be related to the participants' report of their experience of emotional intensity at study, and to long-term memory for that experience. The central questions addressed by this study were (1) whether the amygdala

This article is published in The Journal of Neuroscience, Rapid Communications Section, which publishes brief, peerreviewed papers online, not in print. Rapid Communications are posted online approximately one month earlier than they would appear if printed. They are listed in the Table of Contents of the next open issue of JNeurosci. Cite this article as: JNeurosci, 2000, 20:RC99 (1-5). The publication date is the date of posting online at www.jneurosci.org.

http://www.jneurosci.org/cgi/content/full/4570 
is sensitive to varying degrees of individually experienced emotional intensity and (2) whether the degree of emotional intensity affects the role of the amygdala in enhancing memory for emotional stimuli.

\section{MATERIALS AND METHODS}

Subjects. Ten right-handed healthy female volunteers were scanned in a $1.5 \mathrm{~T}$ magnet. Women were chosen in this study because they are more likely to report intense emotional experiences (Shields, 1991) and show more physiological reactivity in concordance with valence judgments than men (Lang et al., 1993).

Behavioral procedures. During scanning, subjects viewed 96 scenes through a mirror directed at a back-projection screen, each with a normative rating for valence and arousal, from the International Affective Picture System stimuli set (Lang and Greenwald, 1993). For the scenes used in this study, normative ratings for valence ranged from 1.17 (highly negative) to 5.44 (neutral). Normative ratings for arousal ranged from 1.97 (tranquil) to 7.63 (highly arousing). Arousal and valence (degree of negativity) were highly correlated $(r=0.89)$. The order of scenes was randomized across subjects, with each picture presented for a period of $2.88 \mathrm{sec}$. During the interstimulus interval of $12.96 \mathrm{sec}$, subjects viewed a fixation cross. Subjects were instructed to view each picture for the entire time that it was displayed, and after its replacement with a fixation cross they were to indicate their emotional arousal by pressing a button with their right hand. Subjects chose from four buttons to indicate emotional arousal on a scale from 0 ("not emotionally intense at all") to 3 ("extremely emotionally intense"). Three weeks after the scan, subjects were tested in an unexpected recognition test in the laboratory, during which they viewed all of the previously seen and 48 new scenes (foils). The foils were selected to match the previously presented scenes in their valence and arousal characteristics: foils had normative valence ratings that ranged from 1.31 (highly negative) to 5.78 (neutral) and normative arousal ratings that ranged from 2.74 (tranquil) to 7.22 (highly arousing); there was no significant difference between foils and previously presented scenes with respect to their normative valence $\left(t_{(142)}=0.16, p=0.88\right)$ and arousal $\left(t_{(142)}=0.98, p=0.33\right)$ ratings. During the recognition test, subjects were asked whether they had seen each picture before. For scenes judged as previously seen, subjects reported whether they remembered with certainty ("remember") or had a less certain feeling of familiarity ("know"). Forgotten, familiar, and remembered trials were encoded in a numerical format (as 1,2, and 3, respectively) to construct correlation maps.

MRI. Data were acquired in a $1.5 \mathrm{~T}$ General Electric Signa MR imager, which was used to measure blood-oxygen level-dependent (BOLD) contrast (Ogawa et al., 1990). For structural images, eight slices perpendicular to the axial plane of the hippocampus were obtained with a $2.4 \mathrm{~mm}^{2}$ in-plane and $7.0 \mathrm{~mm}$ through-plane resolution. The anterior slice was positioned $7 \mathrm{~mm}$ anterior to the amygdala. Functional images were obtained using a two-dimensional spin echo sequence with two interleaves [repetition time $(\mathrm{TR}) / \mathrm{slice}=90 \mathrm{msec}$, echo time $(\mathrm{TE})=40$ msec, flip angle $=80^{\circ}$, field of view $(\mathrm{FOV})=20 \mathrm{~cm}$, acquisition time $=$ $1.44 \mathrm{sec}$ per frame, number of frames $=264]$. A whole-head coil was used for all subjects. Head movement was minimized by using a bite-bar formed with each subject's dental impression, and motion artifact was examined and corrected automatically for all scans using Air 3.0 (Woods et al., 1992). During functional scanning, 11 frames were captured per trial. Individual frames in each trial were assigned to the baseline fixation period (frames $1,2,10,11$ ) or to the activation period (frames 5-8) based on a lag in peak hemodynamic response of $\sim 4 \mathrm{sec}$ after the presentation of the stimulus (Malonek and Grinwald, 1996).

A correlation map was created to correlate brain activation with subjects' arousal ratings and memory scores as follows: for each trial, the average area under the curve was integrated to measure each voxel's event-related response during both baseline scans and activation scans. Integrated values from baseline scans were then subtracted from activation scans. A Kendal rank-order correlation was calculated for each voxel between event-related responses and reported experienced emotional intensity on a scale from 0 to 3 . Correlation coefficients were transformed into $\mathrm{z}$ scores.

For the composite map shown here, the structural MRI scans were normalized into the same space to allow for the superimposition of statistical maps averaged across subjects onto an averaged structural image. The averaged correlation maps were intensity-thresholded at $p<$ 0.025 , one-tailed, and each slice was subjected to a cluster analysis procedure (Xiong et al., 1995) to correct for multiple statistical comparisons using a spatial extent threshold that yielded a $p<0.025$, one-tailed, significance level over the entire image. Significant voxels located within the region of interest of the amygdala were colored according to their level of significance and overlaid on the averaged structural image.

\section{RESULTS}

Individuals' experience of emotional intensity in the present study correlated well with normative ratings on emotional valence

\section{Degree of Emotional Intensity Correlates with Amygdala Activation}
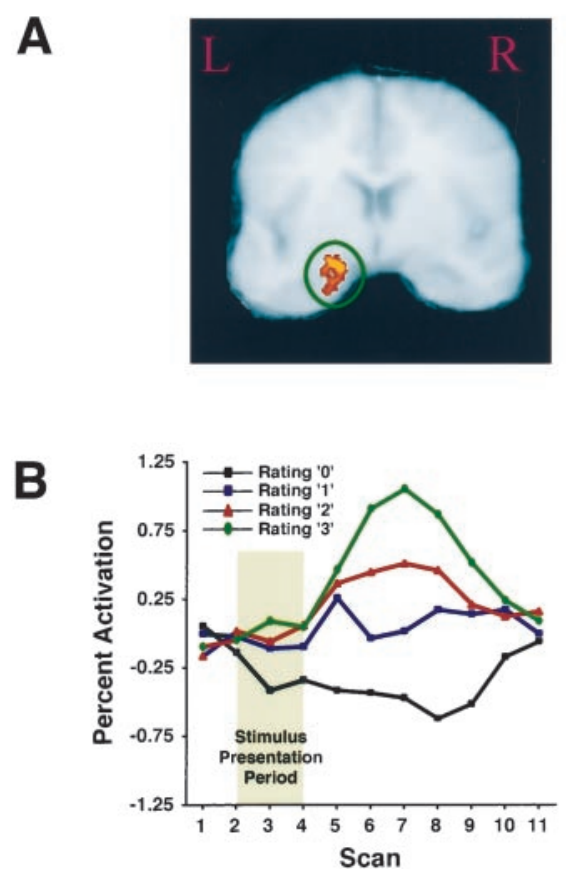

Figure 1. Degree of experienced emotional intensity correlates with amygdala activation. $A$, Cluster of significant correlation between amygdala activation (a priori region of interest) and subjects' emotional intensity ratings. Left side of the image is left side of the brain. Figure shows cluster with Talairach coordinates $-24,-10,-15$, based on Talairach and Tournoux (1988), where $x=$ distance in millimeters to the right $(+)$ or left $(-)$ of midline; $y=$ distance anterior $(+)$ or posterior $(-)$ to the anterior commissure; and $z=$ distance superior $(+)$ or inferior $(-)$ to a horizontal plane through the anterior and posterior commissures. Additional amygdala clusters (data not shown) were located at $+21,-3,-12$, and -28 , $-3,-15 . B$, A time course plot showing average signal intensity in response to scenes that were rated in emotional intensity from 0 (least intense) to 3 (most intense) for the cluster shown in $A$. Averages were made by drawing, for each subject, a region of interest around the left amygdala. Voxels that exhibited significant $(>1.96) z$-scores within this region (representing significant correlations) were averaged for each subject, and then across subjects, for each trial type.

and arousal. The average correlation coefficient between subjects' intensity ratings, on the one hand, and normative valence and arousal, on the other hand, was -0.66 and 0.68 , respectively. Thus, subjects' ratings of emotional intensity reflected equally well the valence and arousal characteristics of the stimuli.

Amygdala activation was significantly, bilaterally correlated with higher ratings of individually experienced emotional intensity (Fig. 1). This represents first evidence that amygdala activation is related to the subjective sense of emotional intensity.

Subjects' ratings of emotional intensity, from 0 to 3 , were similarly distributed across the four intensity categories, with 29, 22,24 , and $25 \%$ of all scenes being rated as $0,1,2$, or 3 , respectively. Therefore, any difference in how well scenes are remembered in a given intensity category (relative to the other categories) cannot be attributed to differences in statistical power. Indeed, scenes in these four categories were not equally well remembered. A repeated-measures ANOVA testing for the effects of reported emotional intensity (ratings from 0 to 3 ) and memory performance (forgotten, familiar, remember) revealed a significant interaction between intensity and memory performance $\left(F_{(6,54)}=3.01, p<0.05\right)$, indicating that memory perfor- 


\section{Enhanced Emotional Memory Correlates With Amygdala Activation To Highly Emotional Stimuli}
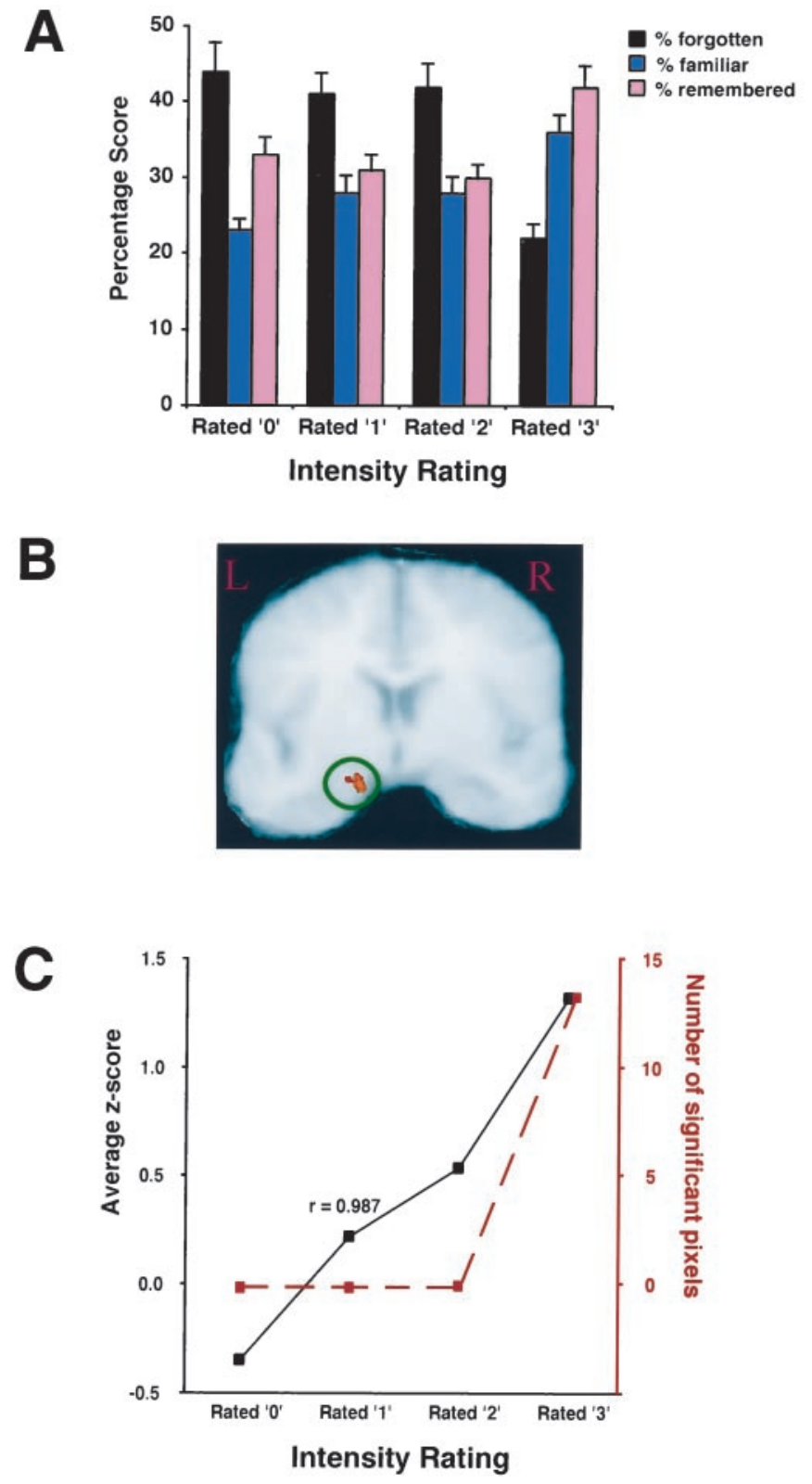

mance was significantly better for scenes that were rated as highly emotionally intense (i.e., rated 3) than for scenes rated less emotionally intense. Scenes that were rated mild-to-moderate (ratings 0 to 2) had similar distributions of items that were forgotten, familiar, or remembered, whereas scenes that were rated as emotionally highly salient (rated 3) were more memorable, because fewer items were forgotten and more were familiar and remembered (Fig. $2 A$ ). The mean false-positive rate for foils was $10 \%$, well below the hit rate for studies scenes, which ranged from 22 to $44 \%$ across these intensity categories.

For scenes that were rated highly emotional (rated 3), the degree of left (but not right) amygdala activation predicted
Figure 2. Enhanced emotional memory correlates with amygdala activation to highly emotionally intense stimuli. $A$, Behavioral data on memory performance as a function of subjects' ratings of emotional intensity. $B$, Cluster of significant correlation between amygdala activation and subjects' memory for scenes rated as emotionally highly intense (rated 3 ). The correlation map was constructed as in Figure 1, except that only trials rated as 3 were included in the analysis, and activation intensity was correlated with three trial outcomes: whether an individual item was forgotten, familiar, or remembered. Left side of the image is left side of the brain. Coordinates of the amygdala are $-20,-10,-14$ (Talairach and Tournoux, 1988). $C$, Correlation between left amygdala activation and memory for emotional items increases with greater emotional intensity. Black solid line shows higher $z$-score averages across the amygdala with higher emotional intensity ratings. $Z$-scores represent the strength of correlation between amygdala activation and subsequent memory for emotional stimuli. The red dotted line shows that there were no pixels within the amygdala that reached statistically significant $z$-score level $(<1.96)$ for scenes that were rated 0 to 2 , but there were 14 significant pixels for scenes that were rated 3 . whether individual stimuli would be forgotten, appear familiar, or be remembered in a later memory test (Fig. 2B). Thus, little amygdala activation in response to a picture rated as highly emotionally intense was associated with the subject's forgetting the stimulus, whereas intermediate and high amygdala activation was associated with a subject's later report of familiarity or confident recognition, respectively.

An additional analysis of the left amygdala revealed a significant correlation between emotional intensity and the modulatory influence of the amygdala on emotional memory. The correlation between amygdala activation and subsequent memory grew stronger as subjects experienced greater emotional intensity (Fig. 2C, 
black line), but only reached significant levels for individual pixels for the most emotionally intense stimuli (Fig. $2 C$, red line). This analysis suggests that a stimulus may need to exceed a certain threshold of emotional intensity before amygdala activation is likely to modulate memory to produce enhanced memory for that stimulus. This is consistent with the behavioral evidence reported in Figure $2 A$ that shows that only the most emotionally intense scenes were associated with superior memory.

Other locations in frontal and temporal regions were noticed that correlated with subjects' individual experiences of emotional intensity and subsequent memory. Because these locations did not constitute a priori regions of interest, they will be considered as tentative constituents of a larger network of structures, until replicated in future studies. The coordinates of these structures are published on our website (http://sucia.stanford.edu/ gablab/ index.shtml).

\section{DISCUSSION}

Event-related fMRI revealed an association between individual experiences of emotional intensity for discrete stimuli with amygdala activation and subsequent memory for these stimuli. This study found that (1) the amygdala is sensitive to individually experienced emotional intensity of discrete visual stimuli; (2) activity in the left amygdala during encoding is predictive of subsequent memory; and (3) the degree to which amygdala activation at encoding can predict subsequent memory is a function of emotional intensity. These findings provide strong new support for the view that amygdala activation reflects moment-to-moment subjective emotional arousal and that this activation enhances memory in relation to the emotional intensity of an experience. These findings are consistent with the hypothesis that the human amygdala enhances declarative memory in a phasic fashion.

The role of the amygdala complex in memory has received considerable attention in recent years. Substantial evidence from studies of both infra-human and human subjects suggests that the amygdala influences the storage of explicit memory for emotionally arousing events (Cahill and McGaugh, 1998). Previous human brain imaging studies showed a correlation between amygdala activation and enhanced declarative memory between subjects, but could not dissociate alternative ("tonic" vs "phasic") interpretations that assigned the amygdala different causal significance.

The hypothesis that the degree of arousal produced by an emotional stimulus determines the degree of amygdala participation in memory storage for the stimulus (Cahill and McGaugh, 1990) predicts that the relationship between amygdala activity at encoding and subsequent memory should become stronger as increasingly arousing stimuli are tested. This important theoretical prediction was not examined in previous imaging studies. In the present study, the correlation between amygdala activity and long-term memory increased significantly as the degree of experienced emotional intensity increased. Whether this correlation was driven by the arousal or valence characteristics of the stimuli cannot be determined, because subjects were only asked to give one rating ("emotional intensity") that correlated equally well with normative valence and arousal ratings. However, the finding of Hamann et al. (1999) that the correlation between amygdala activation and subsequent memory is similar for negative and positive stimuli argues that valence may not be as relevant a determinant as arousal. Future work needs to include emotional stimuli of positive valence with differing arousal ratings and address whether arousal plays a similar role for those stimuli. It is expected that amygdala activation to high arousal positive (or negative) stimuli would be more predictive of subsequent memory than activation to less arousing positive (or negative) stimuli.

In the present study, activity in the left amygdala correlated with subsequent recognition of emotional material. In two PET studies, amygdala activity related to negatively valenced emo- tional stimuli in the right (Cahill et al., 1996) or right-lateralized (Hamann et al., 1999) side correlated with recall. An fMRI study (Canli et al., 1999) reported bilateral amygdala correlations (with larger correlation clusters on the left). One potential explanation of this difference is gender. Both previous PET studies used only male subjects, whereas the previous and current fMRI studies used only females. Laterality could also conceivably relate to differences between the PET and fMRI technologies. Clearly, the determinants of amygdala laterality in influencing memory for emotional events are an important area for future studies. Some of these determinants may be subtle and relate to individual differences in the experience of arousal (Canli et al., 1998), cognitive representations (Phelps et al., 1998), or differences between conscious and unconscious processes (Morris et al., 1998).

Bilateral activity of the amygdala also correlated significantly with the subjects' emotional ratings of the stimuli. One interpretation is that a subject's experience of emotional arousal is the result of amygdala activation, i.e., that the amygdala participates in the production of emotional responses. This interpretation, however, is inconsistent with other data suggesting that the human amygdala is not necessary for the production of emotional responses. Most critically, subjects with amygdala damage often exhibit normal cognitive (Cahill et al., 1995; Adolphs et al., 1997; Hamann et al., 1997) and physiological (Bechara et al., 1995) reactions to emotional stimuli, despite having impaired longterm memory for the stimuli. One subject with bilateral, nearly selective amygdala damage even spontaneously expressed her strong negative emotional reaction to a highly aversive stimulus, but failed to demonstrate enhanced recall of the same stimulus (Adolphs et al., 1997). Finally, the results of a recent study using excitotoxin lesions of the amygdala in monkeys suggest that the emotional changes produced by selective amygdala damage are far smaller than is generally believed (Meunier et al., 1999). Therefore, although the present study shows that subjective experience of emotional intensity is correlated with amygdala activation, other findings imply that the amygdala may not be necessary to experience emotional intensity. Our data suggest that the role of the amygdala is to translate information about the moment-to-moment state of subjective emotional intensity into the modulation of long-term memory, but only for the most emotionally intensive experiences.

\section{REFERENCES}

Adolphs R, Cahill L, Schul R, Babinsky R (1997) Impaired declarative memory for emotional material following bilateral damage in humans. Learn Memory 4:291-300.

Bechara A, Tranel D, Damasio H, Adolphs R, Rockland C, Damasio AR (1995) Double dissociation of conditioning and declarative knowledge relative to the amygdala and hippocampus in humans. Science 269:1115-1118.

Brewer JB, Zhao Z, Desmond JE, Glover GH, Gabrieli JDE (1998) Making memories: brain activity that predicts how well visual experience is remembered. Science 281:1185-1187.

Cahill L, McGaugh JL (1990) Amygdaloid complex lesions differentially affect retention of tasks using appetitive and aversive reinforcement. Behav Neurosci 104:532-543.

Cahill L, McGaugh JL (1998) Mechanisms of emotional arousal and lasting declarative memory. Trends Neurosci 21:294-299.

Cahill L, Babinsky R, Markowitsch HJ, McGaugh JL (1995) The amygdala and emotional memory. Nature 377:295-296.

Cahill L, Haier RJ, Fallon J, Alkire MT, Tang C, Keator D, Wu J, McGaugh JL (1996) Amygdala activity at encoding correlated with long-term, free recall of emotional information. Proc Natl Acad Sci USA 93:8016-8021.

Canli T, Desmond JE, Zhao Z, Glover G, Gabrieli JDE (1998) Hemispheric asymmetry for emotional stimuli detected with fMRI. NeuroReport 9:3233-3239.

Canli T, Zhao Z, Desmond JE, Glover G, Gabrieli JDE (1999) fMRI identifies a network of structures correlated with retention of positive and negative emotional memory. Psychobiology 27:441-452.

Christianson S-A (1992) The handbook of emotion and memory: research and theory. Hillsdale, NJ: Lawrence Erlbaum Associates. 
Hamann SB, Cahill L, Squire LR (1997) Emotional perception and memory in amnesia. Neuropsychology 11:104-113.

Hamann SB, Ely TD, Grafton ST, Kilts CD (1999) Amygdala activity related to enhanced memory for pleasant and aversive stimuli. Nat Neurosci 2:289-293.

Lang PJ, Greenwald MK (1993) International affective picture system standardization procedure and results for affective judgments: technical reports 1A-1C. Center for Research in Psychophysiology. University of Florida, Gainesville.

Lang PJ, Greenwald MK, Bradley MM, Hamm AO (1993) Looking at pictures: affective, facial, visceral, and behavioral reactions. Psychophysiology 30:261-273.

Malonek D, Grinwald A (1996) Interactions between electrical activity and cortical microcirculation revealed by imaging spectroscopy: implications for functional brain mapping. Science 272:551-554.

McGaugh JL, Cahill L, Roozendaal B (1996) Involvement of the amygdala in memory storage: interaction with other brain systems. Proc Natl Acad Sci USA 93:13508-13514.

Meunier M, Bachevalier J, Murray E, Malkova L, Mishkin M (1999) Effects of aspiration versus neurotoxic lesions of the amygdala on emotional responses in monkeys. Eur J Neurosci 11:4403-4418.
Morris JS, Ohman A, Dolan RJ (1998) Conscious and unconscious emotional learning in the human amygdala. Nature 393:467-470.

Ogawa S, Lee TM, Nayak AS, Glynn P (1990) Oxygenation-sensitive contrast in magnetic resonance imaging of rodent brain at high magnetic fields. Magn Reson Med 14:68-78.

Phelps EA, O'Connor KJ, Gatenby JC, Anderson AK, Grillon C, Davis M, Gore JC (1998) Activation of the human amygdala by a cognitive representation of fear: an fMRI study. Soc Neurosci Abstr 24:603.9.

Shields SA (1991) Gender in the psychology of emotion: a selective research review. In: International review of studies on emotion (Strongman KT, ed), pp 227-245. New York: Wiley.

Talairach J, Tournoux P (1988) Co-planar stereotaxic atlas of the human brain. New York: Thieme Medical.

Woods RP, Cherry SR, Mazziotta JC (1992) Rapid automated algorithm for aligning and reslicing PET images. J Comput Assist Tomogr $16: 620-633$.

Xiong J, Gao J-H, Lancaster JL, Fox PT (1995) Clustered pixel analysis for functional MRI activation studies of the human brain. Hum Brain Mapp 3:287-301. 